This is an electronic reprint of the original article. This reprint may differ from the original in pagination and typographic detail.

\author{
Author(s): Doncel, M.; Bäck, T.; Qi, C.; Cullen, D. M.; Hodge, D.; Cederwall, B.; Taylor, M. J.; \\ Procter, M.; Giles, M.; Auranen, Kalle; Grahn, Tuomas; Greenlees, Paul; Jakobsson, \\ Ulrika; Julin, Rauno; Juutinen, Sakari; Herzan, Andrej; Konki, Joonas; Pakarinen, Janne; \\ Partanen, Jari; Peura, Pauli; Rahkila, Panu; Ruotsalainen, Panu; Sandzelius, Mikael; \\ Sarén, Jan; Scholey, Catherine; Sorri, Juha; Stolze, Sanna; Uusitalo, Juha
}

Title: $\quad$ Spin-dependent evolution of collectivity in 112Te

Year: $\quad 2017$

Version:

Please cite the original version:

Doncel, M., Bäck, T., Qi, C., Cullen, D. M., Hodge, D., Cederwall, B., Taylor, M. J., Procter, M., Giles, M., Auranen, K., Grahn, T., Greenlees, P., Jakobsson, U., Julin, R., Juutinen, S., Herzan, A., Konki, J., Pakarinen, J., Partanen, J., . . U Uusitalo, J. (2017). Spin-dependent evolution of collectivity in 112Te. Physical Review C, 96(5), Article 051304(R). https://doi.org/10.1103/physrevc.96.051304

All material supplied via JYX is protected by copyright and other intellectual property rights, and duplication or sale of all or part of any of the repository collections is not permitted, except that material may be duplicated by you for your research use or educational purposes in electronic or print form. You must obtain permission for any other use. Electronic or print copies may not be offered, whether for sale or otherwise to anyone who is not an authorised user. 


\title{
Spin-dependent evolution of collectivity in ${ }^{112} \mathrm{Te}$
}

\author{
M. Doncel, ${ }^{1,2}$ T. Bäck, ${ }^{2}$ C. Qi, ${ }^{2}$ D. M. Cullen, ${ }^{3}$ D. Hodge,${ }^{3}$ B. Cederwall, ${ }^{2}$ M. J. Taylor,,${ }^{3,4}$ M. Procter,${ }^{3}$ M. Giles,${ }^{3}$ \\ K. Auranen, ${ }^{5,6}$ T. Grahn, ${ }^{5}$ P. T. Greenlees, ${ }^{5}$ U. Jakobsson, ${ }^{2,5}$ R. Julin, ${ }^{5}$ S. Juutinen, ${ }^{5}$ A. Herzán̆, ${ }^{1,5}$ J. Konki, ${ }^{5}$ J. Pakarinen, ${ }^{5}$ \\ J. Partanen, ${ }^{5}$ P. Peura, ${ }^{5}$ P. Rahkila, ${ }^{5}$ P. Ruotsalainen, ${ }^{5}$ M. Sandzelius,,${ }^{5}$ J. Sarén,${ }^{5}$ C. Scholey, ${ }^{5}$ J. Sorri, ${ }^{5}$ \\ S. Stolze, ${ }^{5}$ and J. Uusitalo \\ ${ }^{1}$ Department of Physics, Oliver Lodge Laboratory, University of Liverpool, Liverpool L69 7ZE, United Kingdom \\ ${ }^{2}$ Royal Institute of Technology, 10691 Stockholm, Sweden \\ ${ }^{3}$ School of Physics and Astronomy, Schuster Laboratory, The University of Manchester, Manchester M13 9PL, United Kingdom \\ ${ }^{4}$ Division of Cancer Sciences, School of Medical Sciences, The University of Manchester, Manchester, M13 9PL, United Kingdom \\ ${ }^{5}$ University of Jyväskylä, Department of Physics P.O. Box 35, FI-40014 University of Jyväskylä, Finland \\ ${ }^{6}$ Argonne National Laboratory, 9700 South Cass Avenue, Lemont, Illinois 60439, USA
}

(Received 28 August 2017; published 27 November 2017)

\begin{abstract}
The evolution of collectivity with spin along the yrast line in the neutron-deficient nucleus ${ }^{112} \mathrm{Te}$ has been studied by measuring the reduced transition probability of excited states in the yrast band. In particular, the lifetimes of the $4^{+}$and $6^{+}$excited states have been determined by using the recoil distance Doppler-shift method. The results are discussed using both large-scale shell-model and total Routhian surface calculations.
\end{abstract}

DOI: 10.1103/PhysRevC.96.051304

Introduction. Understanding how simple and collective behaviors emerge from complex systems has been one of the most fundamental problems in nuclear physics for many years. The even-mass $\mathrm{Cd}$ and $\mathrm{Te}$ isotopes particularly have been extensively studied both experimentally and theoretically in relation to the long expected possible onset of elusive collective vibration [1]. Among the various experimental approaches applied, high-resolution $\gamma$-ray spectroscopy has been very successful in revealing the nuclear structure in terms of level schemes including precise level energies as well as spin and parity values. Today energy spectra of excited states in tellurium isotopes are known within the wide range from ${ }^{105} \mathrm{Te}$ [2] to ${ }^{139} \mathrm{Te}$ [3]. In particular, the level schemes of low-lying excited states of ${ }^{108-124}$ Te show equally spaced vibrational-like patterns. Transition probabilities for the $\gamma$ decays of the lowest excited state(s) in several isotopes have also been explored and such data now ranges from ${ }^{108} \mathrm{Te}$ [4] to ${ }^{136} \mathrm{Te}$ [5]. There are, however, still gaps along the isotopic chain, where no $B(E 2)$ data exist. This is the case in the midshell- and neutron-deficient region, e.g., for ${ }^{110} \mathrm{Te}$ and ${ }^{116} \mathrm{Te}$, and only recently the lifetime of the $2^{+}$state in ${ }^{112} \mathrm{Te}$ has been measured [6].

Different techniques have been employed for the determination of reduced transition probabilities in Te isotopes. While lifetime measurements using the recoil distance Doppler-shift method have been mainly used for the lighter neutron-deficient exotic isotopes such as ${ }^{108} \mathrm{Te},{ }^{112} \mathrm{Te}$, or ${ }^{114} \mathrm{Te}$ nuclei, principally Coulomb excitation studies have been employed for heavier neutron-rich nuclei around $N=82$ [7].

Lifetimes of several excited states in the nuclide ${ }^{114} \mathrm{Te}$ were studied by Möller et al. [8]. The authors pointed out that while the almost equidistant energy spacings of the ground-state band suggest a vibrational-like structure, the deduced $B(E 2)$ values of the yrast band transitions clearly show a nearly constant behavior as a function of spin and hence disagree with the vibrational model predictions, challenging our present understanding of these nuclides in terms of present models based on single-particle excitations and collective motion. Moreover, the ratio between $B\left(E 2: 4^{+} \rightarrow 2^{+}\right)$and $B\left(E 2: 2^{+} \rightarrow 0^{+}\right)$was measured to be smaller than unity, which is very unusual for a collective nuclear motion and cannot be reproduced by existing theoretical models [8-10].

Here, we report on lifetime measurements in the yrast band of the neighboring (even) isotope ${ }^{112} \mathrm{Te}$. We have approached the problem of the collective nature of this nucleus using both large-scale shell-model calculations and total Routhian surface (TRS) calculations in order to study the shape and softness-todeformation in both nuclei.

Experimental details and method. The excited states in ${ }^{112} \mathrm{Te}$ were populated in the ${ }^{58} \mathrm{Ni}\left({ }^{58} \mathrm{Ni}, 4 p\right)$ fusion-evaporation reaction at the Cyclotron Accelerator Laboratory, Department of Physics, University of Jyväskylä (JYFL), Finland. The experimental setup consisted of the high-purity germanium Jurogam II detector array [11,12] coupled to the recoil ion transport unit (RITU) gas-filled recoil separator [13] and to the differential plunger for unbound nuclear states (DPUNS) [14]. The experimental details have been described in Ref. [6].

The lifetimes have been measured following the principles of the recoil distance Doppler-shift (RDDS) method [15-17] by using the differential decay curve method [18]. Two different approaches were used for the lifetime calculation [19]. For the $6^{+}$excited state at $821 \mathrm{keV}$ the standard known $\gamma-\gamma$ coincidence method, used for the determination of the lifetime of the $2^{+}$excited state in Ref. [6], was employed. On the contrary, for the $4^{+}$excited state at $787 \mathrm{keV}$, due to a contamination peak in the spectra when this method is applied, the approach in which a gate on both components (emitted both before and after the degrader) of a higher-lying transition with respect to the transition of interest is performed was used. Here, the feeding and depopulating transitions of interest are fitted and used to determine the state lifetime using the following equation:

$$
\tau_{i}(x)=\frac{\left\{C_{0}^{\infty}, A_{t}^{\infty}\right\}-\frac{\left\{C_{0}^{\infty}, A_{0}^{\infty}\right\}}{\left\{C_{0}^{\infty}, B_{0}^{\infty}\right\}}\left\{C_{0}^{\infty}, B_{t}^{\infty}\right\}}{\frac{d}{d x}\left\{C_{0}^{\infty}, A_{0}^{t}\right\}} \frac{1}{v},
$$




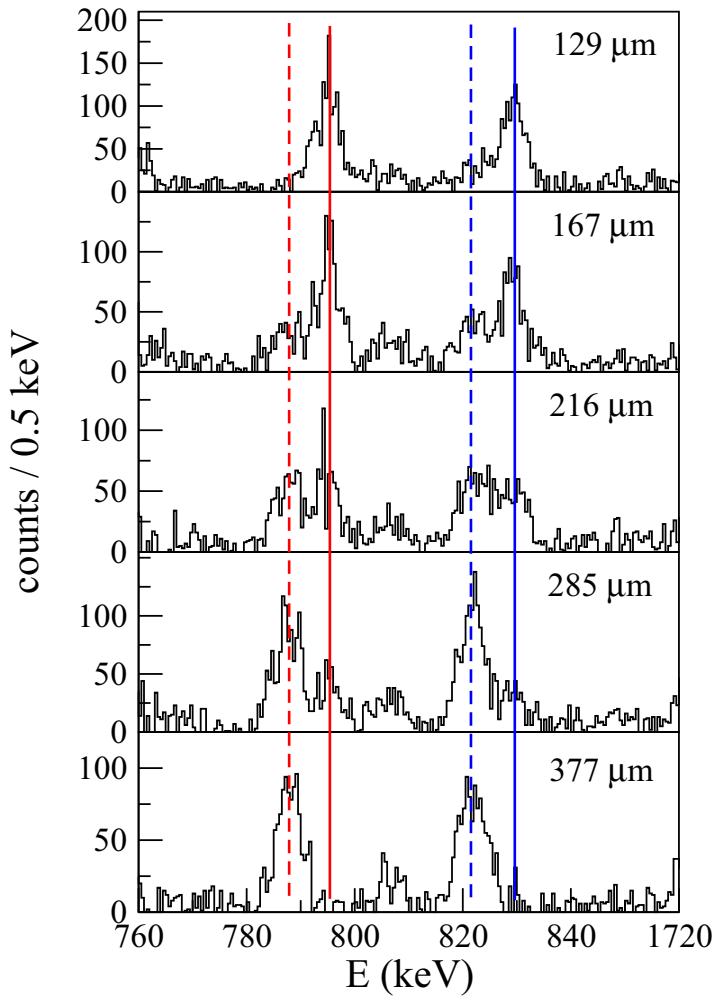

FIG. 1. Spectra obtained after gating on the $18^{+} \rightarrow 16^{+}$transition at $957 \mathrm{keV}$ in ${ }^{112} \mathrm{Te}$ on a $\gamma \gamma$-coincidence matrix for several distances on the sensitivity region where the evolution of the $4^{+} \rightarrow 2^{+}$ (red lines) and the $6^{+} \rightarrow 4^{+}$(blue lines) transitions at 787 and $821 \mathrm{keV}$, respectively, can be observed. Dashed and continuous lines represent the components emitted before and after the degrader, respectively.

where a $\gamma$-ray cascade from $l_{4} \stackrel{C_{\gamma}}{\longrightarrow} l_{3} \stackrel{B_{\gamma}}{\longrightarrow} l_{2} \stackrel{A_{\gamma}}{\longrightarrow} l_{1}$ is considered, with $l_{2}$ being the level of interest. $A_{\gamma}$ and $B_{\gamma}$ stand for the depopulating and feeding transitions, respectively, while $C_{\gamma}$ represents the gating transition. $\left\{C_{0}^{\infty}, A_{t}^{\infty}\right\}$ and $\left\{C_{0}^{\infty}, B_{t}^{\infty}\right\}$ are the intensities of the components produced after the degrader for both transitions $A_{\gamma}$ and $B_{\gamma}$, respectively, when a gate on the $C_{\gamma}$ transition is performed, while $\frac{d}{d x}\left\{C_{0}^{\infty}, A_{0}^{t}\right\}$ corresponds to the slope of the component generated before the degrader for the $A_{\gamma}$ transition when the same gate is applied, determined using the APATHIE code [20]. The expression $\frac{\left\{C_{0}^{\infty}, A_{0}^{\infty}\right\}}{\left\{C_{0}^{\infty}, B_{0}^{\infty}\right\}}$ refers to the ratio between the intensities of the depopulating and the feeding transitions, $A_{\gamma}$ and $B_{\gamma}$, respectively, obtained from the gated spectra. The recoil velocity directly before the degrader was determined to be $v=0.044(1) c$. The intensities of $\gamma$-ray transitions as well as the $\gamma-\gamma$ coincidences were analyzed using the RADWARE data analysis package [21] taking into account detector efficiency as well as internal conversion (coefficients taken from the BrIcc database [22]).

Figure 1 shows the spectra used for the lifetime determination of the $4^{+}$excited state after gating on a higher-lying transition (the $18^{+} \rightarrow 16^{+}$transition at $957 \mathrm{keV}$ for this particular case) for five distances in the sensitivity region. Red and blue colors stand for the $4^{+} \rightarrow 2^{+}$and $6^{+} \rightarrow 4^{+}$ transitions, respectively, while the dashed and continuous

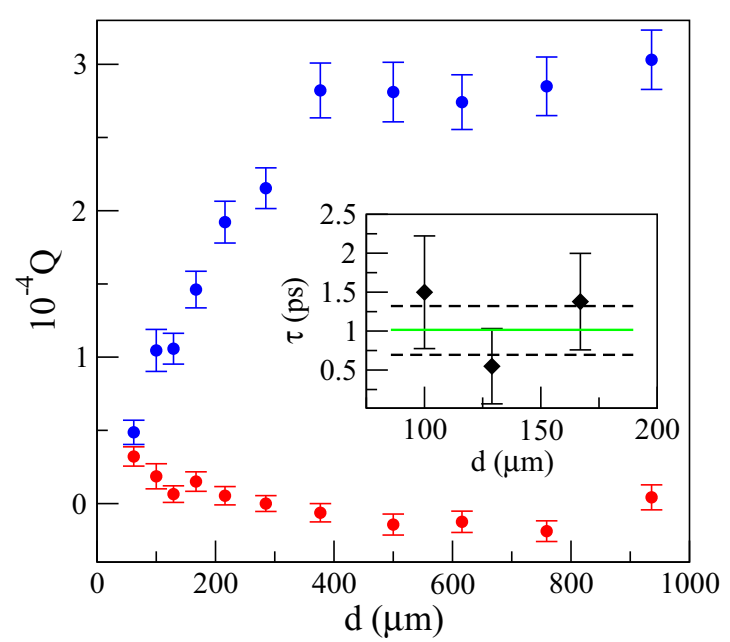

FIG. 2. Normalized intensities of the before and after degrader components (blue and red, respectively) of the $\gamma$ rays depopulating the $6^{+}$excited state, as a function of the distance. The inner panel shows the lifetime value obtained in the present measurement by combining values for three distances in the sensitive region.

lines represent the components before and after the degrader, respectively, for each transition. In Fig. 2 the normalized decay intensities used in the analysis as well as the lifetime value determined for few distances in the sensitivity region (inner caption) for the $6^{+} \rightarrow 4^{+}$transition are shown. The results obtained for the lifetimes of the $4^{+} \rightarrow 2^{+}$and $6^{+} \rightarrow 4^{+}$ transitions are shown in Table I.

It should be noticed that the lifetime values obtained with a gate on the $18^{+} \rightarrow 16^{+}$transition for the $2^{+}$and $6^{+}$ excited states are compatible with the results obtained with the traditional $\gamma-\gamma$ method reported in Ref. [6] and in the present work, respectively.

Discussion. The experimental data on E2 transition probabilities for $\mathrm{Te}$ isotopes have been interpreted within the framework of state-of-the-art shell-model calculations with an optimized realistic interaction within the model space including the single-particle orbitals $g_{7 / 2}, d_{5 / 2}, d_{3 / 2}, s_{1 / 2}$, and $h_{11 / 2}$ between the $N, Z=50$ to 82 shell closures. These are based on our earlier large-scale shell-model calculations as presented in Refs. $[6,10]$. Our calculations can reproduce well the observed equally spaced vibrational-like energy spectra for even-even Te isotopes. The results for ${ }^{112,114} \mathrm{Te}$ are shown in Fig. 3 together with the experimental data. Both the experimental and calculated spectra of the two nuclei show nearly the same

TABLE I. Transition energies $\left(E_{\gamma}\right)$, level lifetimes $(\tau)$, and $B(E 2)$ values for the yrast $2^{+}, 4^{+}$, and $6^{+}$states in ${ }^{112} \mathrm{Te}$. The values for the $4^{+}$and $6^{+}$states are from the present work and for the $2^{+}$state from our earlier study [6].

\begin{tabular}{lccc}
\hline \hline${ }^{112} \mathrm{Te}$ & $E_{\gamma}(\mathrm{keV})$ & $\tau(\mathrm{ps})$ & $B(E 2 \downarrow)_{\text {expt }}($ W.u. $)$ \\
\hline $2^{+}$ & 689 & $5.7(5)$ & $29(3)$ \\
$4^{+}$ & 787 & $1.6(6)$ & $50(20)$ \\
$6^{+}$ & 821 & $1.0(3)$ & $70(20)$ \\
\hline \hline
\end{tabular}



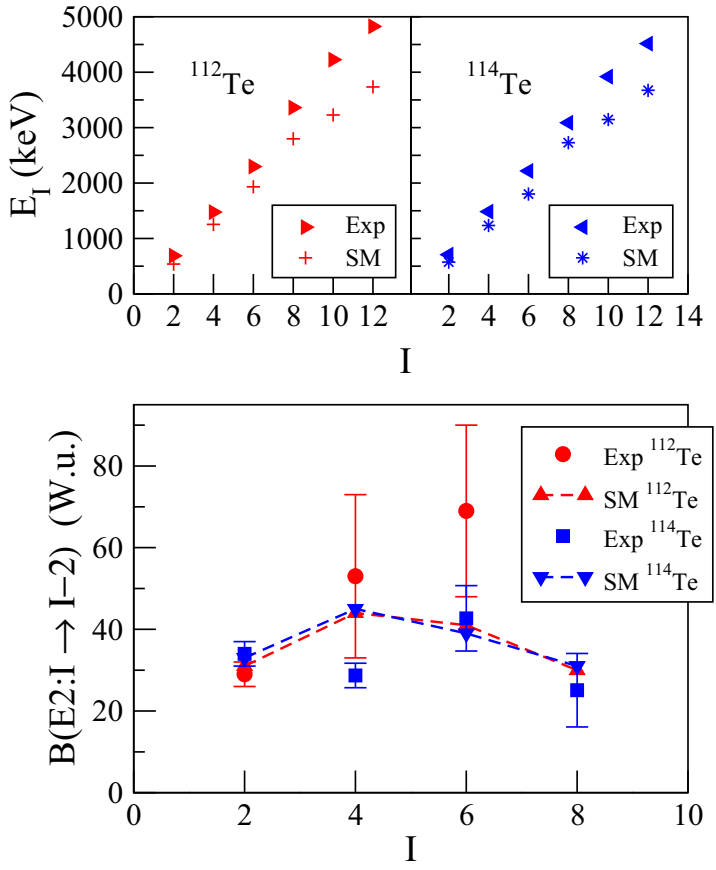

FIG. 3. Top: Experimental values (right and left triangles for ${ }^{112} \mathrm{Te}$ and ${ }^{114} \mathrm{Te}$, respectively) and shell-model calculations for the energies (plus and star for ${ }^{112} \mathrm{Te}$ and ${ }^{114} \mathrm{Te}$, respectively) as a function of spin in the yrast band of ${ }^{112} \mathrm{Te}$ and ${ }^{114} \mathrm{Te}$. Bottom: Experimental values of the reduced transition probabilities as a function of spin for ${ }^{112} \mathrm{Te}$ and ${ }^{114} \mathrm{Te}$ in circles and squares, respectively. The values for ${ }^{114} \mathrm{Te}$ have been taken from [8] while ${ }^{112} \mathrm{Te}$ comes from [6] and from the present work. The up and down triangles correspond to the results obtained with the shell-model calculations from [10]. See text for details.

behavior, indicating that the structure of the nuclei may be quite similar to each other. For these two nuclei we note, however, that the calculations increasingly underestimate the excitation energies of the $8^{+}, 10^{+}$, and $12^{+}$states.

One interesting feature we notice is that the shell-model calculations predict the existence of a second $2^{+}$state which is close to the $4_{1}^{+}$state and a second $4^{+}$state close to the $6_{1}^{+}$ state in both ${ }^{112} \mathrm{Te}$ and ${ }^{114} \mathrm{Te}$. In ${ }^{112} \mathrm{Te}$, the $2_{2}^{+}$and $0_{2}^{+}$states are calculated to be 150 and $240 \mathrm{keV}$, respectively, above the $4_{1}^{+}$state while the $4_{2}^{+}$and $2_{3}^{+}$states are 100 and $225 \mathrm{keV}$, respectively, above the $6_{1}^{+}$state. The $0_{3}^{+}$state is slightly below the $6_{1}^{+}$state. In ${ }^{114} \mathrm{Te}$, the $2_{2}^{+}$and $0_{2}^{+}$states are calculated to be 370 and $430 \mathrm{keV}$, respectively, above the $4_{1}^{+}$state while the $4_{2}^{+}, 0_{3}^{+}$, and $2_{3}^{+}$states are 36,40 , and $260 \mathrm{keV}$, respectively, above the $6_{1}^{+}$state. In the vibrator picture, the second $2^{+}$state close to the $4_{1}^{+}$state is a member of the two-phonon triplet and therefore should have a strong $E 2$ transition to the one-phonon $2_{1}^{+}$state. Similarly, the second $4^{+}$state is then expected to be a member of the three-phonon multiplet. However, in the present calculations, most of those states are weakly connected to the yrast states by $E 2$ transitions. Moreover, a possible mixture between the calculated yrast and yrare $2^{+}$states may significantly reduce the $E 2$ transition strength from the $4_{2}^{+}$ state, which may be the case in ${ }^{114} \mathrm{Te}$.

Two anomalous behaviors in ${ }^{114} \mathrm{Te}$ have been highlighted in Refs. [8,9]: First, the $B\left(E 2: 4^{+} \rightarrow 2^{+}\right)$value is smaller than the $B\left(E 2: 2^{+} \rightarrow 0^{+}\right)$value. Moreover, in contrast to that of a vibrator, the $B(E 2: I \rightarrow I-2)$ values show a nearly constant behavior as a function of spin, which actually is similar to that of a rotor. However, as can be seen from the bottom of Fig. 3, we here observe $B(E 2)$ values for the low-lying yrast states of ${ }^{112} \mathrm{Te}$ that increase as a function of spin.

To understand the different patterns in ${ }^{112} \mathrm{Te}$ and ${ }^{114} \mathrm{Te}$, respectively, we have performed a systematic study of the $B(E 2)$ values in even-even Te isotopes and extracted the ratios $B\left(E 2: 4^{+} \rightarrow 2^{+}\right) / B\left(E 2: 2^{+} \rightarrow 0^{+}\right)$and $B\left(E 2: 6^{+} \rightarrow\right.$ $\left.4^{+}\right) / B\left(E 2: 2^{+} \rightarrow 0^{+}\right)$(denoted as $B_{4 / 2}$ and $B_{6 / 2}$, respectively, in Table II). We notice that ${ }^{112}$ Te shows a behavior which is similar to ${ }^{118,120} \mathrm{Te}$, which are considered to be good examples of quadrupole vibrators and follow the predictions of the $U(5)$ symmetry (vibrator limit) [23-25]. Hence, our results indicate that ${ }^{112} \mathrm{Te}$ also belongs to the same family of vibrational-like systems as ${ }^{118,120} \mathrm{Te}$, which makes the ${ }^{114} \mathrm{Te}$ "anomaly" even more difficult to explain.

We have carried out potential-energy surface (PES) calculations for the ground-state deformations of ${ }^{112} \mathrm{Te}$ and ${ }^{114} \mathrm{Te}$ within the macroscopic-microscopic framework using the Woods-Saxon single-particle potential described in Ref. [26], which is optimized for light- and intermediate-mass nuclei. There is no significant difference between the results for ${ }^{112} \mathrm{Te}$ and ${ }^{114} \mathrm{Te}$ from the PES calculation except for the fact that the ground state of the nucleus ${ }^{114} \mathrm{Te}$ is predicted to have a larger $\beta_{2}$ deformation and be less $\gamma$ soft, as can be seen in Fig. 4. This may be in line with the observation that ${ }^{114}$ Te shows less significant vibrational-like features. Both calculations are dominated by the coupling of protons and neutrons within $g_{7 / 2}$ and $d_{5 / 2}$ orbitals. Moreover, the experimental and calculated ratios between the energies of the $4^{+}$and $2^{+}$states in those two nuclei are both around 2.1, which is closer to that of a quadrupole vibrator (2) than what is expected for a $\gamma$-soft rotor (2.5).

In addition to investigating the ground-state deformation we also performed TRS calculations [27] for both ${ }^{112} \mathrm{Te}$ and ${ }^{114} \mathrm{Te}$ at higher rotational frequencies to see the expected

TABLE II. Experimental $B(E 2)$ values for the $2^{+} \rightarrow 0^{+}, 4^{+} \rightarrow 2^{+}$, and $6^{+} \rightarrow 4^{+}$transitions and $B_{4 / 2}$ and $B_{6 / 2}$ ratios for ${ }^{112}$ Te (present work and taken from Ref. [6] for the $2^{+}$excited state), ${ }^{114} \mathrm{Te}$ [8], ${ }^{118} \mathrm{Te}$ [23], and ${ }^{120,122,124} \mathrm{Te}[25]$.

\begin{tabular}{lccccc}
\hline \hline & ${ }^{112} \mathrm{Te}$ & ${ }^{114} \mathrm{Te}$ & ${ }^{118} \mathrm{Te}$ & ${ }^{120} \mathrm{Te}$ & ${ }^{122} \mathrm{Te}$ \\
\hline$B\left(E 2: 2^{+} \rightarrow 0^{+}\right)_{\text {expt }}$ (W.u.) & $29(3)$ & $34(3)$ & $33(5)$ & $38(1)$ & $36.7(3)$ \\
$B\left(E 2: 4^{+} \rightarrow 2^{+}\right.$)expt (W.u.) & $53(20)$ & $29(3)$ & $70(10)$ & $60(10)$ & $55(2)$ \\
$B\left(E 2: 6^{+} \rightarrow 4^{+}\right.$)expt (W.u.) & $70(20)$ & $43(8)$ & $80(10)$ & $90(20)$ & $30.6(3)$ \\
$B_{4 / 2}$ & $1.8(7)$ & $0.84(8)$ & $2.1(5)$ & $1.64(3)$ & $1.500(40)$ \\
$B_{6 / 2}$ & $2.4(8)$ & $1.3(3)$ & $2.5(5)$ & $2.4(6)$ & $1.162(53)$ \\
\hline \hline
\end{tabular}



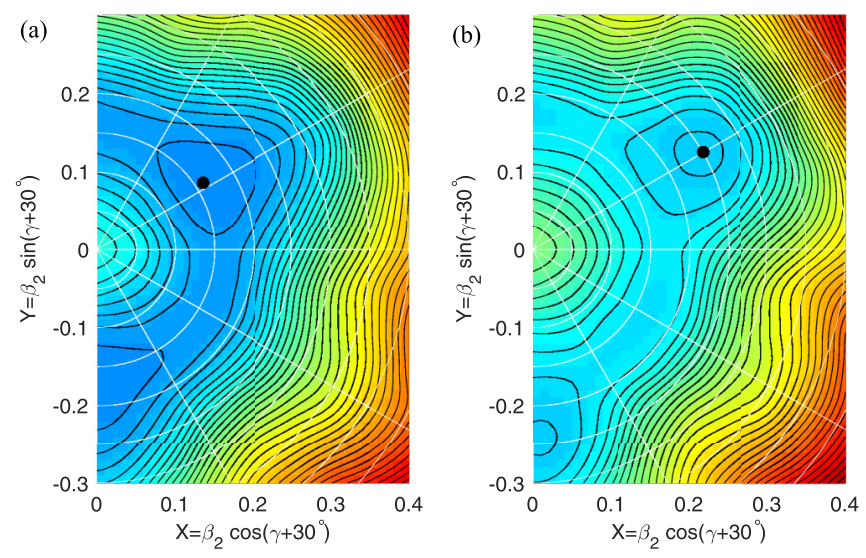

FIG. 4. Potential-energy surface calculations performed with the Woods-Saxon parameters defined in Ref. [26] for (a) ${ }^{112} \mathrm{Te}$ and (b) ${ }^{114} \mathrm{Te}$. The black points correspond to the ground-state deformation minimum.

behavior as we go up to higher spins in the yrast band. These calculations used the cranked shell-model framework with the same parameters as above. Compared to that at the ground state, the prolate energy minimum became more pronounced, indicating a more rigid shape with increased rotational frequency. The results for both nuclides were quite similar. A measurement of the $E 2$ transitions connecting nonyrast $0^{+}$and $2^{+}$states may be necessary to determine the effect of $\gamma$ softness on the collective behaviors of ${ }^{112,114} \mathrm{Te}$.

Summary. The evolution of collectivity as a function of spin has been studied for the neutron-deficient nucleus ${ }^{112} \mathrm{Te}$ along the yrast line. Values of the reduced transition probability for the $4^{+}$and $6^{+}$excited states have been determined by using the recoil distance Doppler-shift method.

Our results indicate that ${ }^{112} \mathrm{Te}$ exhibits vibrational-like patterns similar to what is found in the well-known vibrators ${ }^{118,120} \mathrm{Te}$, as opposed to the anomalous behavior observed in ${ }^{114} \mathrm{Te}$ : The $B\left(E 2: 4^{+} \rightarrow 2^{+}\right)$value is larger than that of $B\left(E 2: 2^{+} \rightarrow 0^{+}\right)$. In addition, the $B(E 2: I \rightarrow I-2)$ values show an increasing trend as a function of spin. However, due to the large uncertainty in the experimental values, a firm conclusion about the collective nature of this nucleus needs further investigation. The shell-model calculations reproduce well the $B(E 2)$ values for the $4^{+} \rightarrow 2^{+}$and $2^{+} \rightarrow 0^{+}$transitions but seem to underestimate the value for the $6^{+} \rightarrow 4^{+}$transition. It is noteworthy that neither the shell-model calculations nor the potential-energy surface calculations performed in this work predicted any significant difference between the structures of ${ }^{112} \mathrm{Te}$ and ${ }^{114} \mathrm{Te}$.

Acknowledgments. The calculations were performed on resources provided by the Swedish National Infrastructure for Computing (SNIC) at NSC in Linköping and PDC at $\mathrm{KTH}$, Stockholm. The authors acknowledge the support of GAMMAPOOL for the loan of the Jurogam detectors. This work was supported by the Swedish Research Council under Grants No. 826-2013-2109, No. 621-2012-3805, and No. 6212013-4323, by the Gran Gustafsson Foundation, by the EU 7th Framework Program, "Integrating Activities Transnational Access," Project No. 262010 (ENSAR), by the Academy of Finland under the Finnish Centre of Excellence Program (Nuclear and Accelerator Based Physics Program at JYFL), by the STFC through a standard grant EP/E02551X/1 (DMC \& MJT), a rolling grant ST/J000159/1, and a consolidated grant ST/L005794/1.
[1] P. E. Garrett and J. L. Wood, J. Phys. G: Nucl. Part. Phys. 37, 064028 (2010).

[2] S. N. Liddick et al., Phys. Rev. Lett. 97, 082501 (2006).

[3] W. Urban et al., Phys. Rev. C 62, 044315 (2000).

[4] T. Bäck et al., Phys. Rev. C 84, 041306 (2011).

[5] D. C. Radford et al., Phys. Rev. Lett. 88, 222501 (2002).

[6] M. Doncel et al., Phys. Rev. C 91, 061304(R) (2015).

[7] M. Danchev et al., Phys. Rev. C 84, 061306(R) (2011).

[8] O. Möller et al., Phys. Rev. C 71, 064324 (2005).

[9] R. B. Cakirli, R. F. Casten, J. Jolie, and N. Warr, Phys. Rev. C 70, 047302 (2004).

[10] C. Qi, Phys. Rev. C 94, 034310 (2016).

[11] F. Beck et al., Prog. Part. Nucl. Phys. 28, 443 (1992).

[12] C. Beausang and J. Simpson, J. Phys. G 22, 527 (1996).

[13] M. Leino et al., Nucl. Instrum. Methods Phys. Res. B 99, 653 (1995).

[14] M. J. Taylor et al., Nucl. Instrum. Methods Phys. Res. A 707, 143 (2013).

[15] T. K. Alexander and J. S. Foster, Adv. Nucl. Phys. 10, 197 (1978).
[16] P. Petkov et al., Nucl. Instrum. Methods. Phys. Res. A 431, 208 (1999).

[17] P. Petkov et al., Nucl. Instrum. Methods. Phys. Res. A 560, 564 (2006).

[18] A. Dewald, S. Harissopulos, and P. Brentano, Z. Phys. A 334, 163 (1989).

[19] A. Dewald et al., Prog. Part. Nucl. Phys. 67, 786 (2012).

[20] F. Seiffert, Program APATHIE, Institut für Kernphysik, Universitat zu Köln, 1989 (unpublished).

[21] D. C. Radford et al., Nucl. Instrum. Methods Phys. Res. Sect. A 361, 297 (1995).

[22] T. Kibedi et al., Nucl. Instrum. Methods Phys. Res. Sect. B 589, 202 (2008).

[23] A. Pasternak et al., Eur. Phys. J. A 13, 435 (2002).

[24] J. R. Vanhoy et al., Phys. Rev. C 68, 034315 (2003).

[25] M. Saxena et al., Phys. Rev. C 90, 024316 (2014).

[26] Z. X. Xu and C. Qi, Phys. Lett. B 724, 247 (2013).

[27] W. Nazarewicz, R. Wyss and A. Johnson, Nucl. Phys. A 503, 285 (1989). 